\title{
Tratamento farmacológico da gagueira: evidências e controvérsias
}

\author{
Pharmacologic treatment of stuttering: evidences and controversies
}

\author{
Camila Vila-Nova, Fernanda Queirós, Thiago Fortaleza e Rita Lucena
}

\begin{abstract}
Resumo
Objetivo: Este artigo tem por objetivo analisar a situação do tratamento farmacológico da gagueira, mostrando a eficácia de diferentes abordagens baseadas em drogas psiquiátricas, além de evidenciar a utilização de outros fármacos no tratamento dessa enfermidade. Métodos: Revisão de literatura em base de dados Medline, utilizando os termos stuttering treatment, disfluency, disfluency treatments, botulinum toxin and stuttering treatment, botulinum toxin and disfluency treatment. Resultados: Foram encontrados estudos envolvendo as seguintes drogas: citalopram + clomipramina, paroxetina, olanzapina, citalopram + alprazolam, pimozida, risperidona, tiaprida, clomipramina e desipramina, levetiracetam, divalproato de sódio, clonidina e betanecol, além de ensaios clínicos com a utilização de toxina botulínica tipo $\mathrm{A}$ e anestésicos. Os estudos envolvendo citalopram + clomipramina, paroxetina, olanzapina, citalopram + alprazolam, risperidona, clomipramina e desipramina, levetiracetam, divalproato de sódio, lidocaína e toxina botulínica tipo A demonstraram resultados positivos. A maioria das pesquisas relativas ao tratamento farmacológico da gagueira se restringe a estudos de caso e ensaios clínicos com pequenas amostras. Conclusão: Não existem evidências suficientes que justifiquem a utilização de um tratamento específico para a gagueira. Os estudos apresentados indicam a necessidade da realização de mais ensaios clínicos duplo-cegos e controlados com placebo envolvendo amostras maiores.
\end{abstract}

Palavras-chave: gagueira, tratamento farmacológico, desordem motora, vias dopaminérgicas.

\begin{abstract}
Objective: This article analyzes the pharmacologic treatment of stuttering, assessing the effectiveness of different treatments using psychiatric drugs and further evidences of other drugs in the treatment of this disorder. Methods: Search in Medline database, using the terms stuttering treatment, disfluency, disfluency treatments, botulinum toxin and stuttering treatment, botulinum toxin and disfluency treatment. Results: Studies involving the following drugs were found: citalopram + clomipramine, desipramine, paroxetine, olanzapine, pimozide, risperidone, tiapride, levetiracetam, divalproex sodium, citalopram + alprazolam, clonidine and bethanecol, as well as clinical trials with the botulinum toxin A and anesthetics. Studies with citalopram + clomipramine, paroxetine, olanzapine, citalopram + alprazolam, risperidone, clomipramine and desipramine, levetiracetam, divalproex sodium, lidocaine and botulinum toxin A showed positive results. However, the great majority of pharmacological studies in this area are case series or clinical trials with small samples. Conclusion: Enough evidences do not exist that justify the use of a specific treatment for stuttering. The presented studies indicate the necessity of accomplishment of more double-blind placebo-controlled trials involving larger samples.
\end{abstract}

Key words: stuttering, pharmacologic treatment, motor disorder, dopamine ways.

Universidade Federal da Bahia (UFBA) (Vila-Nova C, Queirós F, Fortaleza T, Lucena R)

Recebido

25-07-06

Aprovado

26-09-06
Correspondência para: Camila Vila-Nova

Rua Guadalajara, 430/501 - Morro do Gato - Ondina - 40140-461 - Salvador-BA - Tel.: (71) 3235-3802/8827-1325 e-mail: cvnova@gmail.com 


\section{Introdução}

A gagueira é definida como transtorno de fluência (Canevini, 2002) caracterizado pela ocorrência freqüente de um ou mais dos seguintes sinais: repetição de sons, de sílabas ou palavras inteiras, prolongamento de sons, interjeição, quebra na produção de palavra - bloqueios ou interrupções (Gordon, 2002). Pode ainda apresentar circunlóquios e palavras produzidas com tensão física excessiva (Lavid et al., 1999). Há um segundo componente caracterizado pela ansiedade antecipatória, resultado das situações específicas de fala. Essa ansiedade desencadeia bloqueios na fala, tremores de lábios e mandíbula, movimentos palpebrais rápidos, movimentos bruscos de cabeça, braços e tronco superior (Brady e Ali, 2000).

Estudos recentes relatam que a gagueira é um transtorno motor da fala caracterizado por falta de sincronismo entre língua, lábios, laringe e músculos respiratórios (Brin et al., 1994). Esse transtorno é classificado em duas formas: a gagueira do desenvolvimento e a gagueira adquirida ou neurogênica. A primeira, mais comum, tem início na infância ou na adolescência (Canevini, 2002) e pode ou não estar associada a transtornos psiquiátricos. Ocorre no período de desenvolvimento mais significativo da fala e da linguagem e não é secundária a dano cerebral adquirido (Costa e Kroll, 2000). A alteração pode desaparecer no período da puberdade, mas também pode persistir até a vida adulta (Gordon, 2002). A de origem neurogênica é resultado de controle motor anormal (Canevini, 2002).

A gagueira do desenvolvimento predomina no sexo masculino e tem incidência de 1\% a 4\% em crianças (Rosenfield, 2000 apud Gordon, 2002). Estudo realizado pela American Psychiatric Association mostrou prevalência de 2\% em 1.879 estudantes universitários (quarta revisão do Manual Diagnóstico e Estatístico de Transtornos Mentais [DSM-IV]).

Até o momento não existe consenso sobre os fatores etiológicos da gagueira. Fatores psicológicos (Andrews et al., 1983 apud Boldrini, 2003), lesões encefálicas estruturais (Gordon, 2002), disfunção dopaminérgica (Brin et al., 1994), condições neurodegenerativas e fatores genéticos têm sido implicados (Gordon, 2002; Boldrini, 2003). Algumas investigações sobre a neurofisiopatologia têm sugerido hiperatividade nos sistemas dopaminérgicos nas regiões relacionadas à linguagem. Drogas que estimulam a liberação de dopamina podem induzir a ocorrência de gagueira (Lavid et al., 1999). Alguns autores comparam a gagueira com movimentos involuntários distônicos - disfonia espasmódica -, sugerindo a hipótese de o transtorno ser uma forma segmentar ou focal de ação distônica. Sugere-se também que a gagueira seja reflexo de dominância lateral anômala ou conexão inter-hemisférica atípica (Nass, 1996). O papel de fatores genéticos tem sido amplamente reconhecido. A taxa de concordância de ocorrência de gagueira entre gêmeos monozigóticos e dizigóticos é de $90 \%$ e $20 \%$, respectivamente (Gordon, 2002).

Ainda não há protocolo específico para o tratamento farmacológico da gagueira, e os ensaios terapêuticos raramente mostram resultados benéficos em longo prazo (Brin et al., 1994). Este artigo analisa a situação do tratamento farmacológico da gagueira, discutindo a eficácia de diferentes abordagens baseadas em drogas psiquiátricas e a explicação para a escolha de cada uma delas, além de evidenciar a utilização de outros fármacos no tratamento da enfermidade.

\section{Método}

Foi realizada uma revisão em literatura médica especializada - base de dados Medline - entre os anos de 1980 e 2005. Foram utilizados os seguintes termos: stuttering treatment, disfluency, disfluency treatments, botulinum toxin and stuttering treatment, botulinum toxin and disfluency treatment. Incluiram-se estudos controlados e não-controlados. Todos os artigos utilizados foram escritos na língua inglesa. Algumas referências das fontes pesquisadas também serviram como base para o presente estudo.

\section{Tratamento}

Várias drogas têm sido rotineiramente empregadas no tratamento da gagueira (Tabela), entre as quais destacam-se agentes antipsicóticos, benzodiazepínicos, inibidores seletivos de recaptação de serotonina (ISRS) e antidepressivos tricíclicos.

O citalopram, um ISRS, é citado no tratamento da gagueira como droga que não deve ser utilizada isoladamente, pois uma minoria apresenta melhora significativa. Contudo, em estudo de três casos com duração de 20 semanas, observou-se melhora dos sintomas da gagueira quando a droga (10mg ao dia e $20 \mathrm{mg}$ ao dia) foi utilizada em conjunto com o alprazolan (1 $\mathrm{mg}$ duas vezes ao dia) $\mathrm{e}$ com a clomipramina ( $100 \mathrm{mg}$ ao dia). Nos três casos foram relatados poucos efeitos colaterais (Brady e Ali, 2000).

A paroxetina, bloqueador seletivo de recaptação de serotonina, utilizada para tratar o transtorno obsessivo-compulsivo (TOC), foi relatada em um estudo de caso tendo a gagueira como fator de co-morbidade. Observou-se melhora tanto do transtorno psiquiátrico quanto dos sintomas da gagueira depois de quatro meses de tratamento, sendo utilizada uma dose inicial de $20 \mathrm{mg}$ ao dia e, depois, de $30 \mathrm{mg}$ ao dia. $O$ estudo não descreveu efeitos colaterais (Murray e Newman, 1997).

São relatados estudos com o uso de drogas antipsicóticas no tratamento da gagueira em indivíduos sem história de doença psiquiátrica. Uma dessas drogas é a pimozida, que foi utilizada em ensaio clínico controlado em indivíduos gagos e sem sintomas psiquiátricos. Esse estudo evidenciou que $50 \%$ da amostra desenvolveu sintomas de depressão, apesar da diminuição dos sintomas da gagueira (Bloch et al., 1997).

A risperidona causa bloqueio concomitante dos receptores de dopamina $\mathrm{D} 2$ dos núcleos da base e de receptores de serotonina (5-HT2). Um estudo de caso-controle realizado com 16 indivíduos com gagueira, com administração de 0,5 a $2 \mathrm{mg}$ ao dia de risperidona durante seis semanas, mostrou diminuição da gravidade da gagueira sem causar eventos adversos (Maguire et al., 2000). Os mesmos autores desenvolveram um ensaio clínico, randomizado, duplo-cego, placebo-controlado, com 24 pacientes para avaliar a eficácia da olanzapina (dose 2,5 até $5 \mathrm{mg} / \mathrm{dia}$ ) no tratamento da gagueira do desenvolvimento com duração de 20 semanas. A droga mostrou-se significativamente superior ao placebo (Maguire et al., 2004).

Um ensaio clínico placebo-controlado duplo-cego comparou a utilização de clomipramina e desipramina - antidepressivos tricíclicos - no tratamento da gagueira em 16 indivíduos durante 12 semanas, utilizando dose máxima de $250 \mathrm{mg}$ ao dia. Os autores afirmam a superioridade da primeira sobre a segunda. Doze pacientes apresentaram melhora com 0 uso da clomipramina e seis, com o uso da desipramina. 0 estudo ainda relaciona 0 
Tabela. Estudos sobre tratamento farmacológico da gagueira

\begin{tabular}{|c|c|c|c|c|c|c|c|}
\hline Autores & $\begin{array}{l}\text { Ano da } \\
\text { publicação }\end{array}$ & $\begin{array}{l}\text { Número de } \\
\text { pacientes }\end{array}$ & Tipo de estudo & $\begin{array}{l}\text { Droga } \\
\text { utilizada }\end{array}$ & Dose & $\begin{array}{l}\text { Tempo } \\
\text { de tratamento }\end{array}$ & Resultado \\
\hline Althaus et al. & 1995 & 25 & $\begin{array}{l}\text { Ensaio clínico } \\
\text { controlado } \\
\text { cruzado }\end{array}$ & Clonidina & $4 \mu \mathrm{g} / \mathrm{kg} / \mathrm{dia}$ & 28 semanas & $\begin{array}{l}\text { Não houve diferença } \\
\text { entre a droga e o } \\
\text { placebo }\end{array}$ \\
\hline Bloch et al. & 1997 & 8 & $\begin{array}{l}\text { Ensaio clínico } \\
\text { controlado }\end{array}$ & Pimozida & Não informada & 6 semanas & $\begin{array}{l}\text { Diminuição da gagueira, } \\
\text { mas } 4 \text { indivíduos } \\
\text { desenvolveram } \\
\text { sintomas de depressão }\end{array}$ \\
\hline Boldrini et al. & 2003 & 1 & Estudo de caso & Paroxetina & $\begin{array}{l}20 \mathrm{mg} / \mathrm{dia} \\
\left(1 .{ }^{\circ} \mathrm{mês}\right) \\
30 \mathrm{mg} / \mathrm{dia} \\
(12 \mathrm{meses})\end{array}$ & 12 meses & $\begin{array}{l}\text { Diminuiç̧ão da } \\
\text { freqüência e } \\
\text { persistência da gagueira }\end{array}$ \\
\hline Brady \& Ali. & 2000 & 3 & $\begin{array}{l}\text { Descrição de } \\
\text { casos }\end{array}$ & $\begin{array}{l}\text { Alprazolam, } \\
\text { Citalopram and } \\
\text { Clomipramina }\end{array}$ & $\begin{array}{l}\text { Alprazolam } \\
2.0 \mathrm{mg} / \mathrm{dia} \\
\text { Citalopram } 10 \mathrm{a} \\
20 \mathrm{mg} / \mathrm{dia} \\
\text { Clomipramina } \\
100 \mathrm{mg} / \mathrm{dia}\end{array}$ & 20 semanas & $\begin{array}{l}\text { Redução da gagueira e } \\
\text { da ansiedade }\end{array}$ \\
\hline Brin et al. & 1994 & 14 & Ensaio clínico & BTX-A & $1,25 \mathrm{U}$ & 12 semanas & $\begin{array}{l}\text { Melhora moderada da } \\
\text { fluência }\end{array}$ \\
\hline Cavenini et al. & 2002 & 1 & Estudo de caso & Levetiracetam & Não informada & 12 semanas & $\begin{array}{l}\text { Desaparecimento da } \\
\text { gagueira e de convulsões }\end{array}$ \\
\hline Dworkin et al. & 2002 & 1 & Estudo de caso & Lidocaína & 4 cc de $4 \%$ & 12 meses & $\begin{array}{l}\text { Desaparecimento da } \\
\text { gagueira }\end{array}$ \\
\hline $\begin{array}{l}\text { Kampman \& } \\
\text { Brady }\end{array}$ & 1993 & 10 & $\begin{array}{l}\text { Ensaio clínico } \\
\text { controlado } \\
\text { cruzado }\end{array}$ & Betanecol & $30 \mathrm{mg} / \mathrm{dia}$ & 12 meses & $\begin{array}{l}\text { Não foi demonstrada } \\
\text { superioridade da droga } \\
\text { comparada ao placebo. }\end{array}$ \\
\hline Ludlow. & 1990 & 7 & $\begin{array}{l}\text { Ensaio clínico } \\
\text { aberto }\end{array}$ & BTX-A & 10 a $78 U$ & 4 meses & $\begin{array}{l}\text { Significativa redução da } \\
\text { gagueira }\end{array}$ \\
\hline Maguire et al. & 2004 & 24 & $\begin{array}{l}\text { Ensaio clínico } \\
\text { controlado }\end{array}$ & Olanzepina & 2,5 a $5,0 \mathrm{mg} / \mathrm{dia}$ & 20 semanas & $\begin{array}{l}\text { Melhora da gagueira } \\
\text { superior ao placebo }\end{array}$ \\
\hline \multicolumn{8}{|l|}{ Maguire et al. } \\
\hline & 2000 & 16 & $\begin{array}{l}\text { Ensaio clínico } \\
\text { controlado }\end{array}$ & Risperidona & 0,5 a 2,0 mg/dia & 6 semanas & $\begin{array}{l}\text { Melhora significativa da } \\
\text { gagueira superior ao } \\
\text { placebo }\end{array}$ \\
\hline $\begin{array}{l}\text { Mulder \& } \\
\text { Spierrings }\end{array}$ & 2003 & 1 & Estudo de caso & $\begin{array}{l}\text { divalproato de } \\
\text { sódio }\end{array}$ & $1 \mathrm{~g} / \mathrm{dia}$ & 6 anos & $\begin{array}{l}\text { Melhora significativa da } \\
\text { gagueira durante o uso } \\
\text { do medicamento }\end{array}$ \\
\hline $\begin{array}{l}\text { Murray \& } \\
\text { Newman }\end{array}$ & 1997 & 1 & Estudo de caso & Paroxetina & $\begin{array}{l}20 \mathrm{mg} / \mathrm{dia} \\
30 \mathrm{mg} / \mathrm{dia}\end{array}$ & 4 meses & $\begin{array}{l}\text { Melhora dos sintomas } \\
\text { de TOC e da gagueira }\end{array}$ \\
\hline Stager et al. & 1995 & 16 & $\begin{array}{l}\text { Ensaio clínico } \\
\text { controlado } \\
\text { cruzado }\end{array}$ & $\begin{array}{l}\text { clomipramina e } \\
\text { desipramina }\end{array}$ & $250 \mathrm{mg} / \mathrm{dia}$ & 12 semanas & $\begin{array}{l}\text { Demonstrada } \\
\text { superioridade } \\
\text { da clomipramina }\end{array}$ \\
\hline
\end{tabular}

efeito à maior seletividade da droga na inibição de recaptação de serotonina (Stager et al., 1995).

Em estudo de caso foi utilizado o levetiracetam para o tratamento de crises epilépticas em paciente com gagueira do desenvolvimento associada. Observaram-se diminuição da freqüência de crises epilépticas e desaparecimento da gagueira após 12 semanas de tratamento. Os autores justificam esse fato a partir da possível ação do levetiracetam no metabolismo em áreas cerebrais relacionadas à linguagem. Contudo o estudo não revelou a dose utilizada nem se houve ocorrência de efeitos adversos (Canevini et al., 2002).

A eficácia do divalproato de sódio no tratamento da gagueira adquirida é relatada em um caso de paciente com crises epiléticas. A droga foi utilizada na dose de $500 \mathrm{mg}$ duas vezes ao dia entre os anos de 1995 e 1999. Seu uso foi interrompido e retomado em 2001, com seguimento do paciente até 0 ano de 2002. Observou-se melhora da gagueira, contudo sem seu desaparecimento completo durante o uso da medicação. Sedação foi o evento adverso observado (Mulder e Spierings, 2003).

A clonidina utilizada para o tratamento da gagueira infantil aparece em estudo do tipo cruzado, duplo-cego com duração de 28 semanas, envolvendo 25 indivíduos, utilizando a dose de $4 \mu / \mathrm{kg}$ ao dia, no qual não se obteve resultado significante para o tratamento da gagueira (Althaus et al., 1995).

Estudo experimental cruzado envolvendo dez adultos foi desenvolvido durante um ano com o objetivo de mensurar o efeito 
do betanecol sobre a gagueira. Foi administrada a dose de $10 \mathrm{mg}$ três vezes ao dia. Não foi encontrada superioridade dessa droga em relação ao placebo, mas se observou resposta favorável em dois casos. Foi relatada a ocorrência de alguns efeitos colaterais que, entretanto, não foram caracterizados (Kampman e Brady, 1993).

\section{Novas perspectivas}

Estudos que descrevem a ação dos músculos da fala durante a gagueira (Ludlow, 1990) têm demonstrado aumento de atividade, longos períodos de atividade pré-movimento e espasmos de musculatura antagonista de laringe, mandíbula e lábios. Essas características também são encontradas em pacientes com disfonia espasmódica. Por conta disso, foram desenvolvidos ensaios clínicos com a utilização de injeções locais de drogas anestésicas e toxina botulínica do tipo A (BTX-A) na musculatura laríngea para o tratamento da gagueira.

A toxina botulínica é a mais potente neurotoxina biológica conhecida e um grande instrumento na terapia de muitas doenças. Tem sido utilizada para o tratamento de estrabismo, distonias e outras desordens de movimento, além do tratamento da espasticidade. Um número potencial de novas indicações tem emergido e atraído o interesse da comunidade científica. A droga age em uma ou mais proteínas neurossecretoras nas terminações nervosas pré-sinápticas, inibindo a mediação de cálcio para a liberação de acetilcolina na fenda sináptica. Esse fenômeno resulta em denervação química local e perda da atividade neuronal (Thant e Tan, 2003).

Estudo envolvendo sete indivíduos gagos foi realizado para avaliar a eficácia da TBX-A. Os indivíduos foram observados durante o período em que a droga demonstra efeito, ou seja, quatro meses. Foram aplicadas 10 a $78 \mathrm{U}$ de toxina no músculo tireoaritenóideo (TA) esquerdo para provocar paralisia. Significativa redução dos sintomas da gagueira foi detectada, contudo apenas quatro indivíduos repetiram a aplicação. Isso ocorreu devido ao efeito colateral transitório provocado: dispnéia (Ludlow, 1990).

Em outro estudo, 14 pacientes com gagueira do desenvolvimento de grau severo e com espasmos adutores que obstruíam o fluxo aéreo glótico foram submetidos a injeções de 1,25U de TBX-A em cada prega vocal e observados durante 12 semanas. Os benefícios descritos pelos pacientes foram observados na sexta semana após a aplicação, mas o efeito do tratamento diminuiu na $12^{\mathrm{a}}$ semana. Nesse estudo foram observadas fraqueza da voz e disfagia de grau moderado, bem toleradas pelos pacientes, sem desenvolvimento de bronquite ou aspiração (Brin et al., 1994).

Os resultados encontrados nesses estudos podem ser explicados como uma resposta secundária do sistema nervoso à diminuição de atividade muscular após a aplicação de TBX-A. O nível de atividade muscular elevado pode levar a feedback aumentado no tronco cerebral e, conseqüentemente, exacerbar a estimulação dos motoneurônios da laringe, provocando um ciclo repetitivo. A injeção de toxina botulínica reduz o número de fibras que serão ativadas pelo motoneurônio e, em retorno, reduz o feedback de hiperativação do motoneurônio que ocorre no tronco cerebral, criando um efeito de modulação (Ludlow, 1990; Brin et al., 1994).
Recente estudo de caso envolvendo paciente com gagueira adquirida de grau severo também demonstrou melhora significativa com a utilização de anestésico. Lidocaína tópica - 4cc de 4\% - foi administrada na membrana transcricotireóidea. Segundo o relato, a solução anestésica se difundiu para os mecanorreceptores das membranas nas regiões subglótica, glótica e supraglotal. Depois de 15 minutos, a disfluência foi reduzida em $75 \%$, e a velocidade de fala foi de 82 sílabas por minuto. Após uma semana, a redução alcançou $90 \%$, e a taxa de sílaba/minuto foi de 127 . Foram feitos três seguimentos durante o período de um ano. O paciente obteve fluência normal e aumento da velocidade de fala sem recidiva. Não foram descritos efeitos colaterais. Os autores utilizaram a mesma justificativa dos estudos com BTX-A, relatando uma quebra do braço do feedback sensorial (Dworkin et al., 2002).

\section{Considerações sobre os ensaios clínicos envolvendo o tratamento da gagueira}

As dificuldades relativas à caracterização da gagueira refletem-se, conseqüentemente, em uma variedade de propostas de tratamento com o uso de drogas tanto de ação sistêmica como de ISRS, antipsicóticos, antidepressivos tricíclicos, anticonvulsivantes, ansiolíticos, agonistas alfa-2 adrenérgicos, agonistas colinérgicos e drogas de ação tópica (BTX-A e lidocaína). Em alguns estudos os efeitos sobre a gagueira não foram previstos, como no caso daqueles realizados utilizando paroxetina (Murray e Newman, 1997), divalproato de sódio (Mulder e Spierings, 2003) e levetiracetam (Canevini et al., 2002). Isso indica que nem todos os estudos encontrados sobre o tratamento farmacológico da gagueira tiveram como fator primordial a melhora dessa condição.

Diferentes medicamentos foram estudados no tratamento da gagueira. $\mathrm{O}$ estudo de caso com maior seguimento (seis anos) foi um com a utilização do divalproato de sódio (Mulder e Spierrings, 2003); o início do tratamento não foi motivado pela gagueira, que era secundária ao transtorno de base (crises epilépticas). 0 tratamento foi interrompido após quatro anos devido à detecção de deficiência de piruvato quinase, sendo retomado dois anos mais tarde, apesar de ter ficado claro no relato que o divalproato foi prescrito novamente por solicitação do paciente para melhora da gagueira, efeito que havia ocorrido anteriormente. Por outro lado, o maior período de seguimento de ensaios clínicos (12 meses) não foi longo o suficiente para avaliação de recidiva. Alguns relatam efeitos colaterais, como a ocorrência de depressão em indivíduos sem sintomas psiquiátricos (Bloch et al., 1997). A falta de critério rígido para a diferenciação entre gagueira do desenvolvimento e gagueira neurogênica, em grande parte dos estudos envolvendo o tratamento com fármacos, dificulta a compreensão acerca da eficácia das drogas (Gordon, 2002; Dworkin et al., 2002).

Nos estudos que utilizaram injeções locais de TBX-A observou-se também a ocorrência de efeitos colaterais (Ludlow, 1990; Brin et al., 1994). A falta de padronização de dose e sítio de aplicação é uma lacuna que ainda limita a utilização de BTX-A em larga escala no tratamento de gagueira.

Apesar dos diferentes mecanismos de ação, o resultado final encontrado no tratamento da gagueira utilizando psicotrópicos, TBX-A ou anestésicos parece ser o mesmo: a diminuição da atividade muscular. Mas essa hipótese só pode ser considerada com o desenvolvimento de ensaios clínicos controlados com grandes amostras. Muitos dos artigos encontrados em literatura científica 
para o tratamento da gagueira tiveram como alvo a redução da atividade dopaminérgica. Esses resultados podem sugerir a importância do sistema dopaminérgico na fisiopatologia da gagueira, reforçando a hipótese de que há pelo menos um componente fisiológico na origem dessa alteração.

Amaioria das pesquisas relativas ao tratamento farmacológico da gagueira se restringe aos estudos de casos e ensaios clínicos com pequenas amostras. É necessária maior produção de ensaios clínicos controlados com amostras mais significativas.

\section{Conclusão}

Não existem evidências suficientes até o momento que justifiquem a utilização de um tratamento farmacológico específico para a gagueira. Os estudos apresentados indicam a necessidade de realização de ensaios clínicos e duplo-cegos controlados com placebo envolvendo casuística maior e tempo de seguimento prolongado. Ainda são escassos os estudos envolvendo determinadas drogas, o que dificulta 0 avanço das pesquisas no controle de efeitos colaterais.

\section{Referências}

Althaus M, Vink HJ, Minderaa RB, Goorhuis-Brouwer SM, Oosterhoff MD. Lack of effect of clonidine on stuttering in children. Am J Psychiatry, 152: 1087-9, 1995.

American Psychiatric Association. Diagnosis and Statistical Manual of Mental Disorders (DSM-IV). $4^{\text {th }}$ ed. Washington: The Association; 1994.

Bloch M, Stager S, Braun A, Calis KA, Turcasso NM, Grothe DR, Rubinow DR. Pimozide-induced depression in men who stutter. J Clin Psychiatry, 58: 433-6, 1997.

Boldrini M, Rossi M, Placidi GF. Paroxetine efficacy in stuttering treatment. Int J Neuropsychopharmacol, 6: 311-2, 2003.

Borsel JV, Goethals L, Vanryckeghem M. Disfluency in Tourette syndrome: observational study in three cases. Folia Phoniatr Logop, 56: 35866, 2004.

Brady JP, Ali Z. Alprazolam, citalopram and clomipramine for stuttering. J Clin Psychopharmacol, 20: 287, 2000.

Brin MF, Stewart C, Blitzer A, Diamond B. Laryngeal botulinum toxin injections for disabling stuttering in adults. Neurology, 44: 2262-6, 1994.

Cavenini MP, Chiari R, Piazzini A. Improvement of a patient with stuttering on levetiracetam. Neurology, 59: 1288, 2002.

Costa D, Kroll R. Stuttering: an update for physicians. CMAJ, 162: 1849$55,2000$.

Dworkin JP, Meleca RJ. Cullata RA, Abkarian GG. Laryngeal manifestations of speech dysfluency: a topical anesthesia treatment approach. Laryngoscope,112: 936-7, 2002.

Gordon N. Stuttering: incidence and causes. Dev Med Child Neurol, 4: 278-81, 2002

Kampman K, Brady JP. Bethanechol in the treatment of stuttering. J Clin Psychopharmacol, 13: 284-5, 1993.

Lapin I. Phenibut (beta-phenyl-GABA): a tranquilizer and nootropic drug. CNS Drug Rev Winter, 7: 471-81, 2001.

Lavid N, Franklin DL, Maguire G. Management of child and adolescent stuttering with olanzapine: three case reports. Ann Clin Psychiatry, 11: 233-6, 1999.
Ludlow CL. Treatment of speech and voice disorders with botulinum toxin. JAMA, 264: 2671-5, 1990

Maguire GA, Riley GD, Franklin DL, Maguire ME, Nguyen CT, Brojeni $\mathrm{PH}$. Olanzapine in the treatment of developmental stuttering: a double-blind, placebo-controlled trial. Ann Clin Psychiatry, 16: 63-7, 2004

Maguire GA, Riley GD, Franklin DL,Gottchalk LA. Risperidone for the treatment of stuttering. J Clin Psychopharmacol, 20: 479-82, 2000.

Mulder LJ, Spierrings EL. Stuttering relieved by divalproex sodium. Neurology, 6: 311-2, 2003.

Murray MG, Newman RM. Paroxetine for treatment of obsessivecompulsive disorder and comorbid stuttering. Am J Psychiatry, 154: 1037, 1997.

Nass RD. Developmental stutter in a patient with callosal agenesis disappears during steroid therapy. Pediatr Neurol, 15: 1668, 1996.

Prins D, Mandelkon T, Cerf FA. Principal and differential effects of haloperidol and placebo treatments upon speech disfluencies in stutterers. J Speech Hear Res, 23: 614-29, 1980.

Rothenberger A, Johannsen HS, Schulze H, Amorosa H, Rommel D. Use of tiapride on stuttering in children and adolescents. Percept Mot Skills, 79: 1163-70, 1994.

Schreiber S, Pick CG. Paroxetine for secondary stuttering: further interaction of serotonin and dopamine. J Nerv Ment Dis, 185: 465-7, 1997.

Stager SV, Ludlow CL, Gordon CT, Cotelingan M, Rapoport JL. Fluency changes in persons who stutter following a double blind trial of clomipramine and desipramine. J Speech Hear Res, 38: 516-25, 1995.

Steele JW, Faulds D, Sorkin EM. Tiapride. A review of its pharmacodynamic and pharmacokinetic properties, and therapeutic potential in geriatric agitation. Drugs Aging, 3: 460-78, 1993.

Thant ZS, Tan EK. Emerging therapeutic applications of botulinum toxin. Med Sci Monit, 9: RA40-8, 2003. 